\title{
Frequency jump in an ion linac
}

\author{
R. Duperrier, ${ }^{1}$ N. Pichoff, ${ }^{2}$ and D. Uriot ${ }^{1}$ \\ ${ }^{1}$ CEA/Saclay, 91191 Gif sur Yvette cédex, France \\ ${ }^{2}$ CEA/DAM Ile de France, BP 12-91680 Bruyères-le-Châtel, France
}

(Received 8 September 2006; published 1 August 2007)

\begin{abstract}
Frequency jumps in an ion linac use to be made in order to provide a large transverse acceptance in the low-energy part and a high accelerating gradient in the high-energy part. This frequency jump may induce a discontinuity in the average longitudinal force per focusing period and shrink the longitudinal acceptance of the linac if this transition is not performed carefully. In this paper, three techniques are developed which produce a "certain" continuity of the channel at the transition between. The continuity type is discussed. It is demonstrated that the longitudinal acceptance can be preserved whatever the frequencies of the cavities in the linac. This point is very important when comparisons between different cavity types are made (spoke and elliptical cavities for, instance). A few examples are shown to illustrate the performances of the three techniques.
\end{abstract}

DOI: 10.1103/PhysRevSTAB.10.084201

PACS numbers: 29.27.Bd, 29.27.Eg, 29.27.Fh, 41.75.-i

\section{INTRODUCTION}

Frequency jumps in ion linacs use to be made in order to provide a large transverse acceptance (physical aperture) in the low-energy part and a high accelerating gradient and/or a better shunt impedance in the high-energy part. The minimization of the size of the cavities in the high-energy section is also interesting to reduce the cost because these cavities are the most numerous in the accelerator. Smaller cavities help to reduce the cryogenic load and induce a cheaper fabrication. This frequency jump may induce a discontinuity in the average longitudinal force per focusing period and shrink the longitudinal acceptance of the linac if this transition is not performed carefully. In Ref. [1], techniques are shown to manage the transition an radio frequency quadrupole operating at $350 \mathrm{MHz}$ and a coupled cavity drift tube linac (CCDTL) operating at $700 \mathrm{MHz}$. It is explained that as the frequency is doubled in the CCDTL, a conservative synchronous phase of -60 degrees is required at the beginning of the structure to capture all particles. The concept of the continuity of the phase advance per unit length is discussed for the transverse plane in order to provide a current and emittance independent design. This continuity simplifies significantly the matching at the transition. In this paper, we propose to develop techniques for designers to tune the phase and the field at frequency transitions in an ion linac which include acceptance and phase advance per unit length issues. During the European Spallation Source studies in 2000 [2], we developed a first technique to keep constant the confinement potential shape at the frequency jump. The goal was to maintain the beam in the achieved equilibrium state. Later, we proposed a different approach based on the continuity of the acceptance of the system. More recently, a third technique which is a mix of the first two has been proposed.
In this paper, these three techniques are developed and compared to the classical method which is a matching at the transition (tuning of the focusing elements to maintain a smooth evolution of the phase advance per meter in the following section) keeping a high accelerating efficiency.

\section{CONSTANT POTENTIAL SHAPE}

The superconducting option for the European Spallation Source linac is made with different sections which operate at different frequencies $(175,350$, and $700 \mathrm{MHz})$ [3]. To render transparent these frequency jumps to the beam, a first technique to keep the confinement potential shape constant at the transition has been developed [2]. This technique aims at maintaining the beam in the achieved equilibrium state. This point is especially relevant when a very intense beam has to be transported [4]. As the longitudinal force is nonlinear (sine), assuming that the beam has reached an equilibrium in the low frequency section, a continuity of these nonlinearities is mandatory in order to avoid a new emittance growth in the high frequency section. From the equation of the longitudinal motion, it is shown in [5] that the potential well can be written:

$$
\begin{aligned}
V(\delta \phi)= & -\frac{2 \pi}{m(\beta \gamma c)^{3} f_{\mathrm{rf}}} \cdot q E_{0} T \\
& \cdot\left[\cos \phi_{s}(\sin \delta \phi-\delta \phi)+\sin \phi_{s} \cdot(\cos \delta \phi-1)\right],
\end{aligned}
$$

with $\delta \phi$ the phase shift of the particle with respect to the synchronous phase $\phi_{s}, f_{\mathrm{rf}}$ the operating frequency, $\beta$ the reduced speed, $\gamma$ the Lorentz factor, $E_{0} T$ the average field per focusing period taking into account the transit time factor, $c$ the speed of light, $m$ the mass of the particle, and $q$ its charge. Writing $\delta \phi=2 \pi f_{\mathrm{rf}} \delta t$ and developing at third 
order in $\delta t$ the expression (1), one finds

$$
\begin{aligned}
V(\delta t)= & \frac{2 \pi}{m(\beta \gamma c)^{3} f_{\mathrm{rf}}} \cdot q E_{0} T \\
& \cdot\left[\cos \phi_{s} \cdot\left(2 \pi f_{\mathrm{rf}}\right)^{3} \cdot \frac{\delta t^{3}}{6}+\sin \phi_{s} \cdot\left(2 \pi f_{\mathrm{rf}}\right)^{2} \cdot \frac{\delta t^{2}}{2}\right] .
\end{aligned}
$$

The two preceding equations can be used for any axis field profile of the electrical longitudinal component of the cavity if the synchronous phase $\phi_{s}$ is calculated with the definition given in the Appendix. The approach is then relevant for a standing wave cavity with equal or not equal cell length.

At a frequency change, one should try to keep this potential well as continuous as possible. This can be made at third order by changing both the synchronous phase and the accelerating field. One has to solve the equation system:

$$
\left\{\begin{array}{l}
E_{0} T \cdot f_{\mathrm{rf}}^{2} \cdot \cos \phi_{s}=\mathrm{cst} \\
E_{0} T \cdot f_{\mathrm{rf}} \cdot \sin \phi_{s}=\mathrm{cst} .
\end{array}\right.
$$

If $f_{1}$ is the frequency for the section before the transition and $f_{2}$ the frequency for the section after, writing $k=$ $f_{2} / f_{1}$, this gives the necessary conditions:

$$
\left\{\begin{array}{l}
\tan \left(\phi_{s}\right)_{2}=k \cdot \tan \left(\phi_{s}\right)_{1} \\
\left(E_{0} T\right)_{2}=\frac{\left(E_{0} T\right)_{1}}{k^{2}} \sqrt{\frac{1+k^{2} \tan ^{2}\left(\phi_{s}\right)_{1}}{1+\tan ^{2}\left(\phi_{s}\right)_{1}} .} .
\end{array}\right.
$$

The second condition in the system (3) is equivalent to keep the phase advance per meter constant. The relative variation of the accelerating rate can be calculated with the following formula:

$$
\Re=\frac{\left(E_{0} T\right)_{2} \cos \left[\left(\phi_{s}\right)_{2}\right]}{\left(E_{0} T\right)_{1} \cos \left[\left(\phi_{s}\right)_{1}\right]} .
$$

This gives

$$
\Re=\frac{1}{k^{2}} .
$$

If $f_{1}<f_{2}, \Re$ is lower than 1 . This is the main drawback of the method.

\section{CONTINUITY OF THE ACCEPTANCE}

We developed a second approach to calculate the field and the phase at the transition. Instead of fitting the potential shape, the longitudinal acceptance is kept constant. Taking into account that the phase is not a canonical coordinate, this statement implies that $\Delta W$ (energy) and $\Delta Z$ (for the position, and not $\Delta \Phi$ ) have to be maintained at the transition. To compute the expression for $\Delta Z=\beta \lambda$. $\Delta \Phi / 2 \pi$, we use the following approximation for the acceptance in phase,

$$
\Delta \Phi \sim 3 \phi_{s},
$$

where $\phi_{s}$ is the synchronous phase. It is valid when $\phi_{s}$ is close to the crest (strong acceleration) which is the case for our problem. For $\Delta W_{\max }$ conservation, we will use the analytical formula [5]:

$$
\Delta W_{\max }= \pm 2\left[\frac{q m c^{3} \beta^{3} \gamma^{3} E_{0} T\left(\phi_{s} \cos \phi_{s}-\sin \phi_{s}\right)}{f_{\mathrm{rf}}}\right]^{1 / 2} .
$$

This formula is derived from the integration of the motion in the longitudinal plane. The integration is performed with the assumption that the speed is quasiconstant during the acceleration. This assumption is valid at high energy which is, again, relevant for this study. To keep constant $\Delta Z$ and $\Delta W$ at the frequency jump, it is required that

$$
\left\{\begin{array}{l}
\frac{\phi_{s}}{f_{\mathrm{rf}}}=\mathrm{cst} \\
\frac{E_{0} T\left(\phi_{s} \cos \phi_{s}-\sin \phi_{s}\right)}{f_{\mathrm{rf}}}=\mathrm{cst} .
\end{array}\right.
$$

Writing $k=f_{2} / f_{1}$, the first condition gives

$$
\left(\phi_{s}\right)_{2}=k\left(\phi_{s}\right)_{1} \text {. }
$$

For the second condition, replacing the cosine and sine functions by a Taylor expansion at second order (which is justified because we are working close to the crest) and inserting in the system (9) gives

$$
\left(E_{0} T\right)_{2}=\frac{\left(E_{0} T\right)_{1}}{k^{2}} .
$$

It appears that the accelerating rate is also reduced at the transition. The relative reduction of the accelerating rate can be calculated with the following formula:

$$
\Re=\frac{\cos \left[k\left(\Phi_{s}\right)_{1}\right]}{k^{2} \cos \left[\left(\Phi_{s}\right)_{1}\right]} .
$$

If $f_{1}<f_{2}, \mathfrak{R}$ is always lower than 1 . If $\Phi_{s}$ tends to zero, Eq. (12) is equal to Eq. (6).

Using the same Taylor expansions at second order, it can be shown that the conditions (10) and (11) induce a constant phase advance per meter at the transition if $\Phi_{s}$ is sufficiently close to the crest.

One may be surprised that Eq. (2) is derived at third order instead of the second order for Eq. (8). It can be verified that a second order treatment of (2) is not equivalent and produces a poor efficiency [2].

\section{CONTINUITY OF THE PHASE ACCEPTANCE AND THE PHASE ADVANCE PER METER}

Recently, we developed a third approach to perform the transition which is a mix of the two previous ones. We set that the phase acceptance (but not the energy one) and the phase advance per meter have to be kept constant. It implies that 


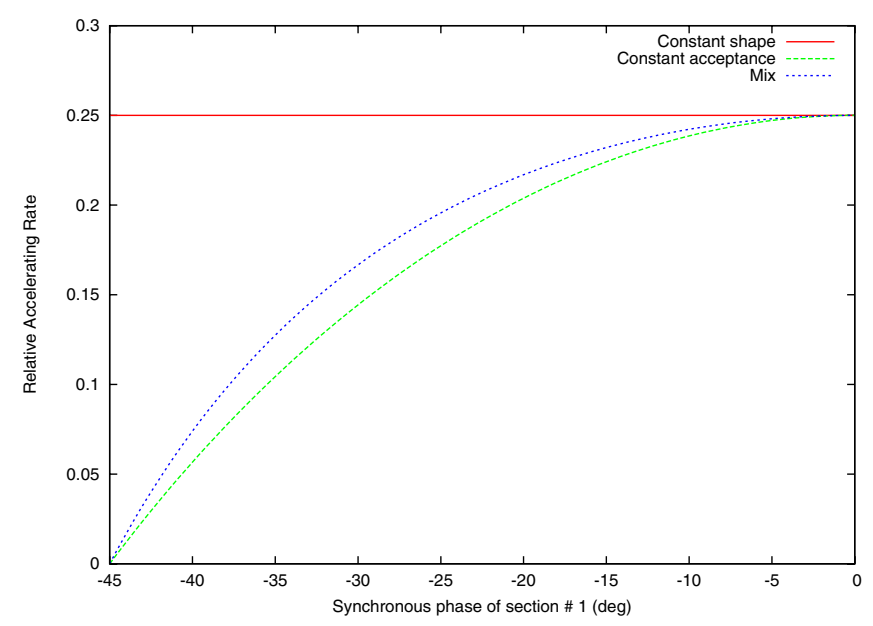

FIG. 1. (Color) The relative accelerating rate reduction with respect to the synchronous phase of the low frequency section when $k=2$.

$$
\left\{\begin{array}{l}
\frac{\phi_{s}}{f_{\mathrm{rf}}}=\mathrm{cst} \\
E_{0} T \cdot f_{\mathrm{rf}} \cdot \sin \phi_{s}=\mathrm{cst} .
\end{array}\right.
$$

Writing again $k=f_{2} / f_{1}$, we found from (13) that the phase and $E_{0} T$ have to follow the relations

$$
\left\{\begin{array}{l}
\left(\phi_{s}\right)_{2}=k \cdot\left(\phi_{s}\right)_{1} \\
\left(E_{0} T\right)_{2}=\frac{\left(E_{0} T\right)_{1}}{k} \frac{\sin \left[\left(\phi_{s}\right)_{1}\right]}{\sin \left[k\left(\phi_{s}\right)_{1}\right]} .
\end{array}\right.
$$

The relative accelerating rate reduction can be calculated with the formula (15):

$$
\mathfrak{R}=\frac{\tan \left[\left(\Phi_{s}\right)_{1}\right]}{k \cdot \tan \left[k\left(\Phi_{s}\right)_{1}\right]} .
$$

A comparison of the 3 accelerating rate (AR) reduction is shown in Fig. 1 for $k=2$ and a synchronous phase of the low frequency section varying from -45 to 0 degrees. It can be noticed that the reduction of the AR can be total for the two last methods.

\section{BENCHMARKS}

To test the efficiency of the three techniques and to illustrate the relevancy of such methods, this section compares the emittance growth induced by an increasing input longitudinal emittance in a test linac. No space charge is taken into account for this test, the nonlinearity of the longitudinal $\mathrm{rf}$ restoring force (sine) is the only source of the emittance growth. In a second part, the relative conservation of the acceptance is computed for different input synchronous phase in the linac.

\section{A. Emittance growth}

One figure of merit for the beam dynamics is the conservation of the initial emittance in the linac. To test our three techniques, we propose to use a section of accelerating periods starting at $50 \mathrm{MeV}$. The lattice is FDO. The accelerating voltage is set to $2 \mathrm{MV}$ and the initial synchronous phase is -20 degrees. The length of the section is 80 meters with 22 focusing periods. The operating frequency of the first half is $352 \mathrm{MHz}$. For this first subsection, the field and synchronous phase are kept constant. We designed 5 versions for the second part of the linac: (i) a constant field and phase linac with a frequency of $352 \mathrm{MHz}$ to get a reference simulation which can be compared to the versions with a frequency jump; (ii) a constant field and phase linac with a frequency of $704 \mathrm{MHz}$ and a matching at the transition (focusing elements are changed around the transition to obtain a smooth evolution of the phase advance per meter in the following section); (iii) a linac at $704 \mathrm{MHz}$ with field and phase held constant per section, the second section obeys the technique "constant acceptance"; (iv) a linac at $704 \mathrm{MHz}$ with field and phase held constant per section, the second section obeys the technique "constant potential shape"; (v) a linac at $704 \mathrm{MHz}$ with field and phase held constant per section, the second section obeys the technique "constant phase acceptance and phase advance per meter." For each of these 5 linacs, we computed several transports increasing the input longitudinal emittance. For each run, the relative emittance growth produced by the nonlinear rf force has been calculated. Figure 2 shows the behavior of these emittance growths. An emittance blowup can be noticed for the linac with just a matching at the transition, whereas the emittance growth behaviors for the three techniques are comparable with the one for the linac which has no frequency jump. No loss has been observed in the simulations to be capable to compare the emittance evolutions. To illustrate the effect in the longitudinal plane of the nonlinear $\mathrm{rf}$ force on a case, Fig. 3 shows the phase space portraits of the beam at the exit of the section for three cases: when one single frequency is used, when the frequency is doubled

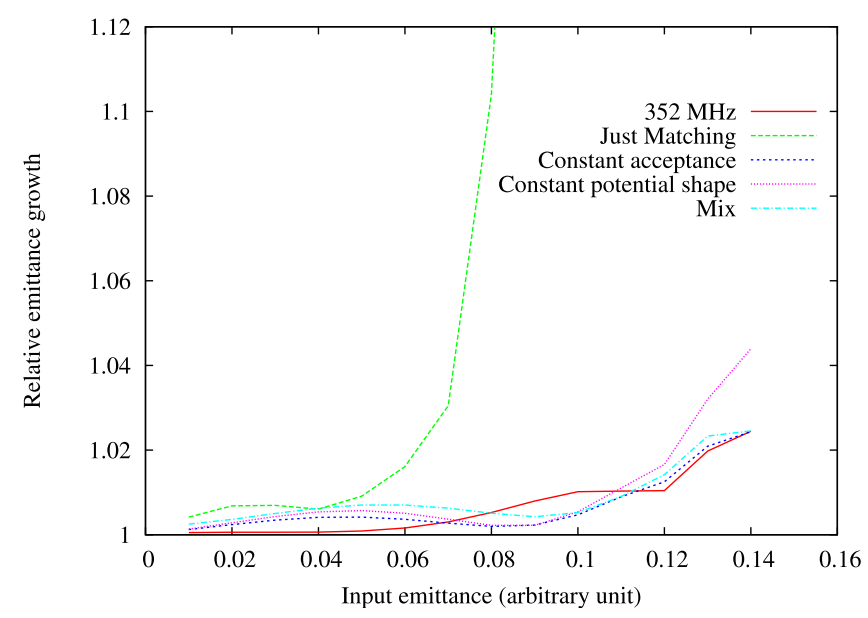

FIG. 2. (Color) The relative emittance growths for an increasing input longitudinal emittance for the four linacs. 
NGOOD : $40000 / 40000 \quad \mathrm{l}=0.0 \mathrm{pA} \quad$ PlotWin - CEA/DSM/DAPNIA/SACM

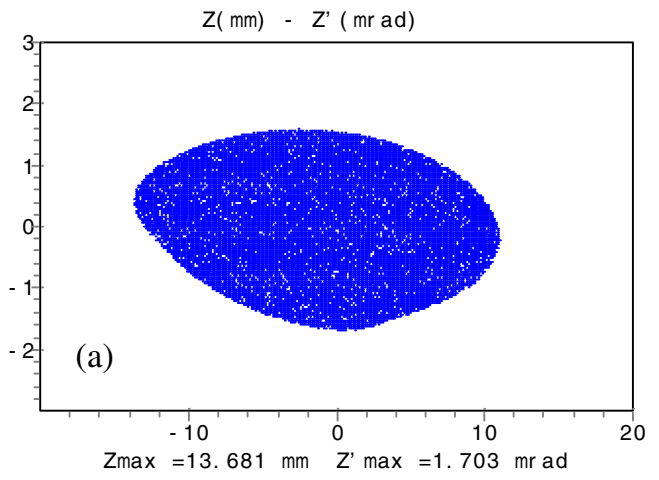

Ele: 222 [79.2 m] NGCOD : $200000 / 200000$ TraceWin -CEA DSM/DAPNIA/SACN
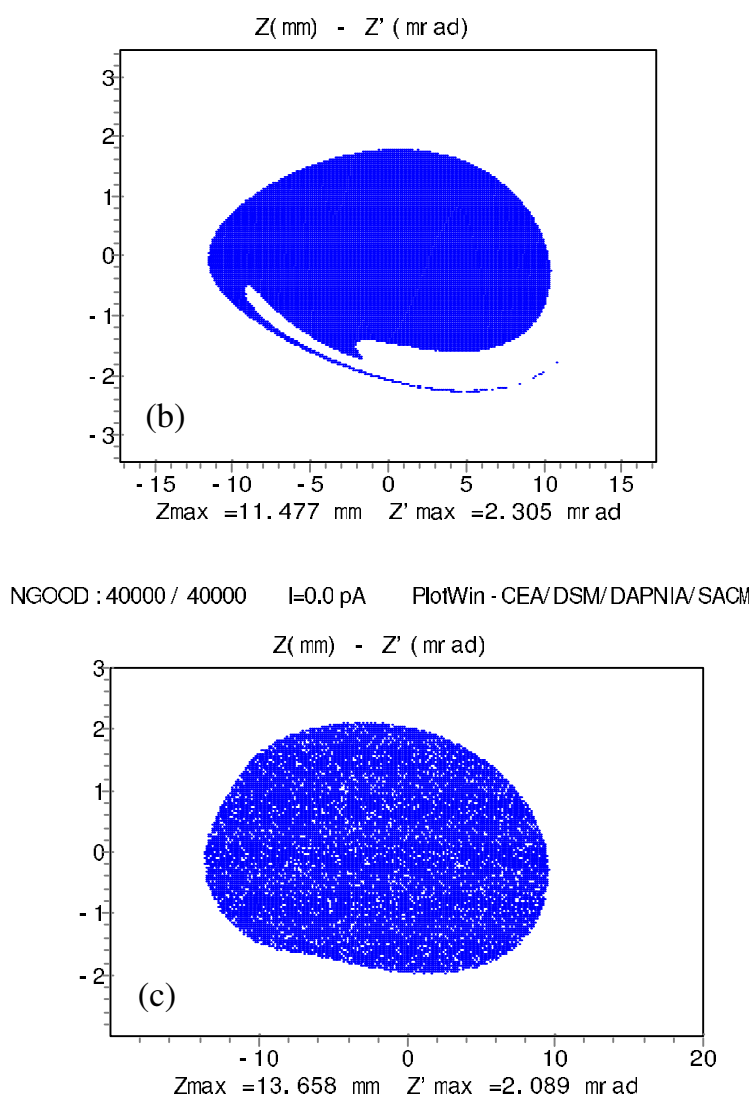

FIG. 3. (Color) Phase space portraits of the beam at the exit for three cases: (a) single frequency $(+0.3 \%)$, (b) frequency jump with a matching at transition $(+2 \%)$, (c) frequency jump the mix technique $(+0.6 \%)$.

and a matching is proceeded, and when the frequency is doubled and the technique "constant phase acceptance and phase advance per meter" is applied. For each case, the relative emittance growth is given. To illustrate the obtained potential well according to the used method, Fig. 4 shows the potential shape for the three different techniques for an initial synchronous phase of -45 degrees which maximizes the discrepancies.

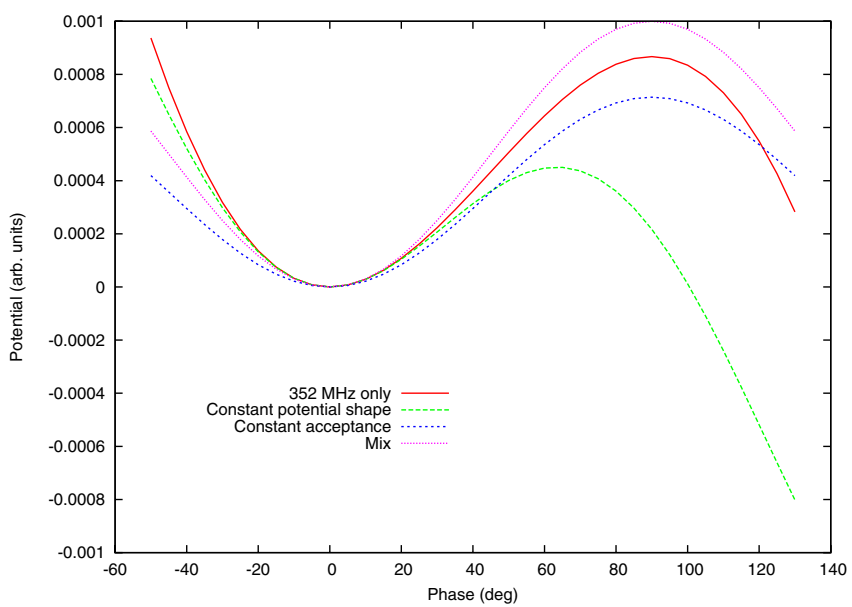

FIG. 4. (Color) The potential well shape for the three different techniques for an initial synchronous phase of -45 degrees.

\section{B. Acceptance}

Several hypothesis have been used to obtain the frequency transition rules in the previous sections. For the first technique, it is assumed that the phase shift between the particles and the synchronous one is small. For the second technique, the hypothesis that the synchronous phase is close to zero is made. For the three techniques, it is assumed that the speed of the particle is constant in an accelerating gap. To check these approximations, we propose to compute the acceptance of the preceding linacs with the following method: (i) simulate the transport of a huge cloud of particles which are initially uniformly distributed in the longitudinal phase space; (ii) then, count the surviving particles to allow a relative comparison of the linac acceptances. The area of the initial distribution in phase space has to be significantly larger than the calculated acceptance. We repeated these simulations for differ-

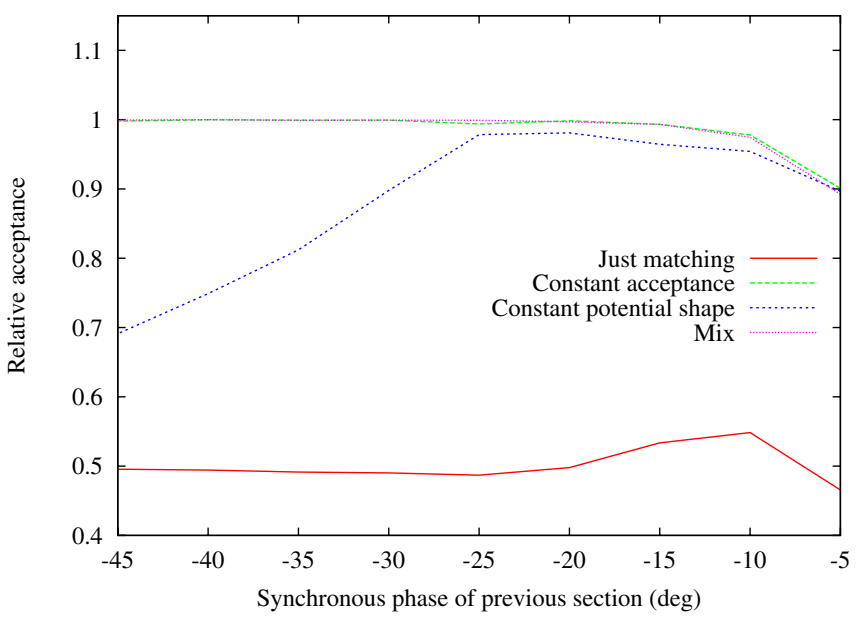

FIG. 5. (Color) The relative acceptance of the linacs with frequency jumps in respect to the initial synchronous phase. The reference is the linac without frequency jump. 
ent initial synchronous phases from -45 degrees to -5 degrees to check the validity of the several approximations about the phase. Figure 5 shows the evolution of

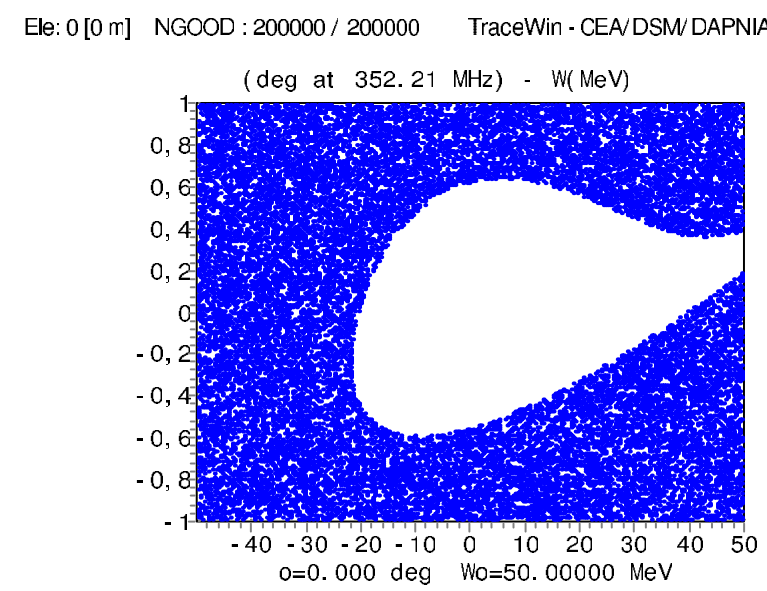

(a)
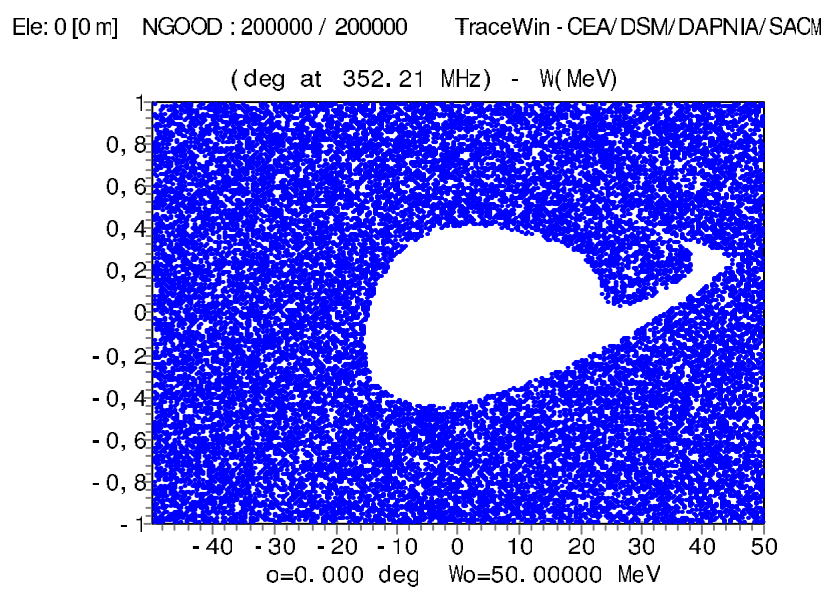

(b)

Ele: 0 [0 m] NGOCD :200000/200000 TraceWin-CEA/DSM/DAPNIA/SACM

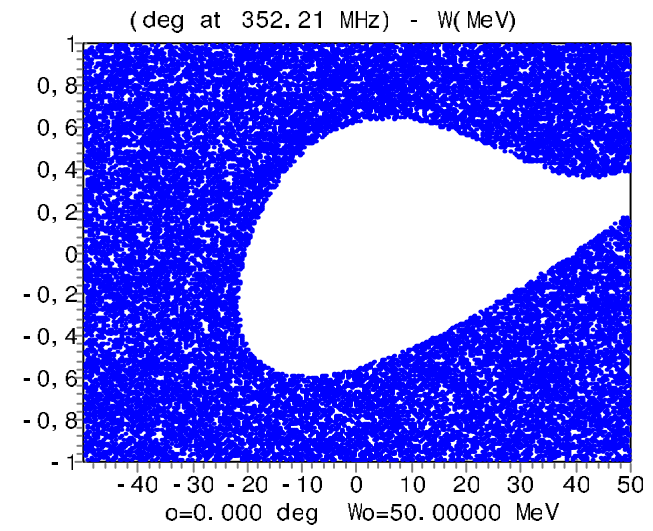

(c)

FIG. 6. (Color) Computed acceptance for three cases: (a) single frequency, (b) frequency jump with a matching at transition, (c) frequency jump the mix technique. the relative acceptances for linacs with a frequency jump with respect to the linac with only $352 \mathrm{MHz}$ cavities.

First, it is clear that keeping constant the field and the phase and performing a matching at the transition is not satisfying. The acceptance is reduced by a factor 2 . Second, the second and the third techniques appear to be very efficient for a wide range of synchronous phase. The acceptance is conserved even if the synchronous phase is relatively far from the crest. Let us recall that the AR tends to zero for such a case. For the technique of the constant potential shape, it is equivalent to the two previous ones in the range beyond -25 degrees to the crest. To illustrate the impact on the acceptance for the different techniques, Fig. 6 shows the computed acceptance in the longitudinal plane for three cases: single frequency, frequency jump with a matching at transition, frequency jump the "mix" technique.

It can be noticed that the efficiency for the three methods slowly decreases when the phase is close to zero. The reason is the approximation of the constant speed for the integration of the motion which provided Eqs. (1), (7), and (8). This approximation is not valid any more when the acceleration is sufficiently high to significantly change the particle speed in a period. For a synchronous phase of -5 degrees, when the field is decreased by a factor 10 in order to minimize the speed change in an accelerating gap, the relative acceptance is increased from 0.9 to 0.96 . Differently, if the input energy is increased by a factor 10 to produce the same effect, the relative acceptance is increased from 0.9 to 0.98 .

\section{CONCLUSIONS}

We investigated three different techniques to manage a frequency change in a ion linac to render it as transparent as possible: the "constant potential shape," the "constant acceptance," and a mix which is a constant phase acceptance and a constant phase advance per meter. We compared them with the common method which is a simple matching maintaining a high acceleration efficiency. It is shown that the three techniques significantly enhanced the acceptance of the system. To simplify and to provide to linac designers rules which are valid for a wide range of parameters, we recommend to use the following formulas at the transition:

$$
\left\{\begin{array}{l}
\left(\phi_{s}\right)_{2}=k\left(\phi_{s}\right)_{1} \\
\left(E_{0} T\right)_{2}=\frac{\left(E_{0} T\right)_{1}}{k} \frac{\sin \left[\left(\phi_{s}\right)_{1}\right]}{\sin \left[k\left(\phi_{s}\right)_{1}\right]} .
\end{array}\right.
$$

with $k=f_{2} / f_{1}, f_{1}$ the frequency for the section before the transition, $f_{2}$ the frequency for the section after, $\left(E_{0} T\right)_{i}$ the average field taking into account the transit time factor, and $\left(\phi_{s}\right)_{i}$ the synchronous phase of the section $i$. Let us note that the continuity of the phase advance per meter is then preserved and the acceptance is not shrunk for a synchronous phase lower than -25 degrees. Moreover, the accel- 
erating rate is slightly better compared to the technique of the "constant acceptance." Nevertheless, in case of an intense beam which reached the equilibrium in the section at low frequency in which a nonlinear focusing force is applied, the first technique has to be preferred. It allows one to maintain the same nonlinearity and then to avoid a new emittance growth [4].

The main drawback of the three methods is a reduction of the accelerating rate at the transition. But the higher the exit energy of the linac, the lower the impact of this reduced acceleration at the transition since the field amplitude can be ramped gradually. It is then possible to enhance significantly the accelerating efficiency with the high gradient of high frequency cavities (warm or superconducting). It is very important to respect such rules at the frequency transition in the linac when comparisons between different cavities are made from the beam dynamics point of view (spoke and elliptical cavities, for instance, in [6]).

\section{APPENDIX: ENERGY GAIN IN A rf CAVITY}

For a rf cavity with a length $L$, the energy gain for a charged particle which traverses the cavity on the axis with an amplitude profile of the longitudinal component $E_{z}(s)$ can be calculated with the formula

$$
\Delta W=\int_{s_{0}}^{s_{0}+L} q E_{z}(s) \cdot \cos [\phi(s)] \cdot d s,
$$

with $q$ the charge of the particle, and $s$ the beam axis coordinate. The function $\phi(s)$ is the rf phase when the particle is at the coordinate $s$. It is defined by

$$
\phi(s)=\phi_{0}+\frac{\omega_{\mathrm{rf}}}{c} \int_{s_{0}}^{s_{0}+s} \frac{d s^{\prime}}{\beta_{z}\left(s^{\prime}\right)}
$$

with $c$ the Einstein constant, $\omega_{\text {rf }}$ the rf pulsation, $\phi_{0}$ is the rf phase when the particle is at the cavity entrance, and $\beta_{z}\left(s^{\prime}\right)$ is the longitudinal component of the particle reduced speed at the $s^{\prime}$ location. Writing $\phi(s)=\phi(s)+\phi_{s}-\phi_{s}$, with $\phi_{s}$ being an arbitrary phase and using trigonometric relations, we found that the energy gain can be written

$$
\begin{aligned}
\Delta W= & \cos \left(\phi_{s}\right) \int_{s_{0}}^{s_{0}+L} q E_{z}(s) \cdot \cos \left[\phi(s)-\phi_{s}\right] \cdot d s \\
& -\sin \left(\phi_{s}\right) \int_{s_{0}}^{s_{0}+L} q E_{z}(s) \cdot \sin \left[\phi(s)-\phi_{s}\right] \cdot d s .
\end{aligned}
$$

We can define $\phi_{s}$ such as

$$
\int_{s_{0}}^{s_{0}+L} q E_{z}(s) \cdot \sin \left[\phi(s)-\phi_{s}\right] \cdot d s=0 .
$$

It gives

$$
\phi_{s}=\arctan \left[\frac{\int_{s_{0}}^{s_{0}+L} q E_{z}(s) \cdot \sin (\phi(s)) \cdot d s}{\int_{s_{0}}^{s_{0}+L} q E_{z}(s) \cdot \cos (\phi(s)) \cdot d s}\right] .
$$

Then the energy gain can be rewritten:

$$
\begin{aligned}
\Delta W & =\left[q \int_{s_{0}}^{s_{0}+L}\left|E_{z}(s)\right| \cdot d s\right] \cdot T \cdot \cos \phi_{s} \\
& =q V_{0} \cdot T \cdot \cos \phi_{s}
\end{aligned}
$$

with

$$
T=\frac{1}{V_{0}} \int_{s_{0}}^{s_{0}+L} q E_{z}(s) \cdot \cos \left[\phi(s)-\phi_{s}\right] \cdot d s .
$$

$T$ is known as the transit time factor. It depends on the speed of the particle and the field amplitude. A crucial point is that no hypothesis about the field profile in the cavity has been made to develop these formulas. This general approach can then be used for a standing wave resonator with a fixed or not fixed geometrical $\beta$. This definition of the synchronous phase is different compared to the one which can be found in the literature which is the rf phase when the particle reaches the middle of the cavity. This definition and our definition correspond exactly when the cavity is symmetric and the speed variation can be neglected. It has to be noticed that, in fixed $\beta$ cavities, there is no synchronism between cells, but this redefinition of the phase allows us to use the classical formalism [5]. This phase can then be called "effective" phase or "reference" phase. With this last hypothesis, the parameter $T$ depends only on the average speed of the particle in the cavity. The calculation of $T$ with Eq. (A7) may be difficult because it is required to know the value of $\phi_{s}$. But when the speed gain is weak enough, this dependence can be neglected and it is possible to use another equation for the transit time factor:

$$
T=\frac{1}{V_{0}}\left|\int_{s_{0}}^{s_{0}+L} q E_{z}(s) \cdot e^{j \phi(s)} \cdot d s\right| .
$$

[1] J. H. Billen, H. Takeda, and L. M. Young, Proceedings of the LINAC-1996 on Smooth Transverse and Longitudinal Focusing in High-Intensity Linacs, p. 587.

[2] N. Pichoff et al., ESS LINAC Technical Note ESSLINTN-1001-04.

[3] The ESS Project, Technical report, Vol. III, 2002.

[4] M. Reiser, J. Appl. Phys. 70, 1919 (1991).

[5] P. Lapostolle and M. Weiss, CERN-PS-2000-001-DR, 2000.

[6] K. W. Shepard, P. N. Ostroumov, and J. R. Delayen, Phys. Rev. ST Accel. Beams 6, 080101 (2003). 